\section{Facharztprüfung zur Erlangung des Facharzttitels FMH für Neurologie}

Aufgrund des Weiterbildungsprogrammes, welches am 1. Juli 1999 in Kraft gesetzt wurde, ist die Teilnahme an der Facharztprüfung für diejenigen Kandidatinnen und Kandidaten Voraussetzung für die Erlangung des Facharzttitels FMH für Neurologie, welche ihre Weiterbildung in Neurologie am 31. Dezember 1996 nicht abgeschlossen hatten. Es empfiehlt sich, die Facharztprüfung frühestens im letzten Jahr der reglementarischen Weiterbildung abzulegen (Art. 22 WBO).
Erster Teil

theoretisch (Multiple choice, auf Englisch)

Ort: CHUV, Lausanne

Zeitpunkt: Donnerstag, 13. Juni 2002, 14.00 Uhr

Zweiter Teil

mündlich und praktisch

Ort und Zeitpunkt: wird später bekanntgegeben (Herbst 2002).

Prüfungsgebühr: Die SNG erhebt eine Prüfungsgebühr von Fr. 1000.-. Die Vorweisung der Quittung des Einzahlungsscheines ist erforderlich für die Zulassung zum theoretischen Teil der Prüfung.

Anmeldung: Schriftliche Anmeldung unter Beilage des Ausbildungscurriculums an: Prof. Dr. med. J. Bogousslavsky, Service de Neurologie, CHUV-BH 13, 1011 Lausanne, Tel. 02131412 20, Fax 0213141231.

Anmeldefrist : Mittwoch, 13. März 2002

\section{Examen de spécialiste en vue de l'obtention du titre FMH en neurologie}

Conformément au programme de formation postgraduée qui est entré en vigueur le $1^{\text {er }}$ juillet 1999 , la participation à l'examen de spécialiste est obligatoire pour les candidats à l'obtention du titre FMH en neurologie qui n'avaient pas terminé leur formation postgraduée en neurologie le 31 décembre 1996. Il est recommandé de passer l'examen de spécialiste au plus tôt durant la dernière année de formation postgraduée réglementaire (art. 22 RFP).
Première partie

partie théorique (multiple choice questionnaire, en anglais)

Lieu: CHUV, Lausanne

Date: le jeudi, 13 juin 2002, 14:00 h

Deuxième partie

partie orale et pratique

Lieu et Date: seront communiqués ultérieurement (automne 2002).

Taxe d'examen: Fr. 1000.- sont prélevés par la Société Suisse de Neurologie. La présentation du récépissé de versement est obligatoire pour être admis à la partie théorique.

Inscription: A adresser par écrit en associant un curriculum de formation à l'adresse suivante: Prof. J. Bogousslavsky, Service de Neurologie, CHUV-BH 13, 1011 Lausanne, tél. 02131412 20, fax 0213141231.

Délai d'inscription: mercredi, 13 mars 2002 